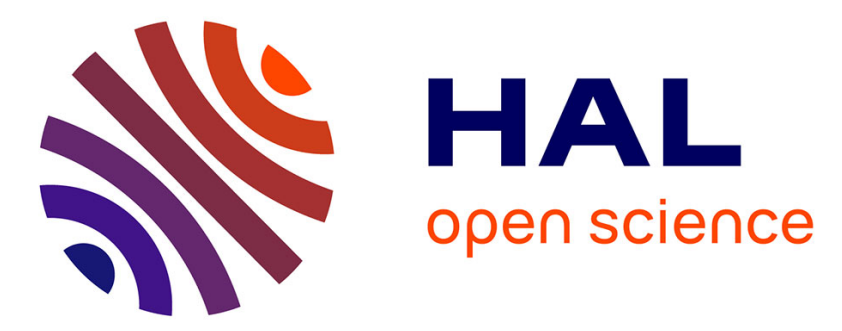

\title{
$\beta$-TCP granules mixed with reticulated hyaluronic acid induce an increase in bone apposition
}

Eric Aguado, Florence Pascaretti-Grizon, Christine Gaudin, Eric Goyenvalle, Daniel Chappard

\section{To cite this version:}

Eric Aguado, Florence Pascaretti-Grizon, Christine Gaudin, Eric Goyenvalle, Daniel Chappard. $\beta$ TCP granules mixed with reticulated hyaluronic acid induce an increase in bone apposition. Biomedical Materials, 2014, 9 (1), pp.015001. 10.1088/1748-6041/9/1/015001 . hal-03265958

\section{HAL Id: hal-03265958 \\ https://univ-angers.hal.science/hal-03265958}

Submitted on 21 Jun 2021

HAL is a multi-disciplinary open access archive for the deposit and dissemination of scientific research documents, whether they are published or not. The documents may come from teaching and research institutions in France or abroad, or from public or private research centers.
L'archive ouverte pluridisciplinaire HAL, est destinée au dépôt et à la diffusion de documents scientifiques de niveau recherche, publiés ou non, émanant des établissements d'enseignement et de recherche français ou étrangers, des laboratoires publics ou privés. 


\title{
$\beta$-TCP granules mixed with reticulated hyaluronic acid induce an increase in bone apposition
}

\author{
Eric Aguado ${ }^{1,2}$, Florence Pascaretti-Grizon ${ }^{2}$, Christine Gaudin-Audrain ${ }^{2}$, \\ Eric Goyenvalle $^{1,2}$ and Daniel Chappard ${ }^{2,3}$ \\ ${ }^{1}$ ONIRIS, Ecole Vétérinaire de Nantes, F-44307 Nantes Cedex 3, France \\ 2 LUNAM Université, GEROM-LHEA 'Bone Remodeling and bioMaterials', IRIS-IBS Institut de \\ Biologie en Santé, CHU d'Angers, F-49933 ANGERS Cedex, France \\ E-mail: daniel.chappard@univ-angers.fr
}

Received 26 February 2013

Accepted for publication 6 September 2013

Published 16 December 2013

\begin{abstract}
$\beta$ beta-tricalcium phosphate ( $\beta$-TCP) granules are suitable for repair of bone defects. They have an osteoconductive effect shortly after implantation. However, dry granules are difficult to handle in the surgical room because of low weight and lack of cohesion. Incorporation of granules in a hydrogel could be a satisfactory solution. We have investigated the use of hyaluronic acid (HyA) as an aqueous binder of the granules. $\beta$-TCP granules were prepared by the polyurethane foam technology. Commercially available linear (LHya) and reticulated hyaluronic acid (RHyA) in aqueous solution were used to prepare a pasty mixture that can be handled more easily than granules alone. Thirteen New Zealand White rabbits $(3.5-3.75 \mathrm{~kg})$ were used; a $4 \mathrm{~mm}$ hole was drilled in each femoral condyle. After flushing, holes were filled with either LHyA, RHyA, dry $\beta$-TCP granules alone, $\beta$-TCP granules + LHyA and $\beta$-TCP granules + RHyA. Rabbits were allowed to heal for one month, sacrificed and femurs were harvested and analysed by microCT and histomorphometry. The net amount of newly formed bone was derived from measurements done after thresholding the microCT images for the material and for the material+bone. LHyA and RHyA did not result in healing of the grafted area. LHyA was rapidly eluted from the grafted zone but allowed deposition of more granules, although the amount of formed bone was not significantly higher than with $\beta$-TCP granules alone. RHyA permitted the deposition of more granules which induced significantly more bone trabeculae without inducing an inflammatory reaction. RHyA appears to be a good vehicle to implant granules of $\beta$-TCP, since HyA does not interfere with bone remodeling.
\end{abstract}

(Some figures may appear in colour only in the online journal)

\section{List of abbreviations}

$\beta$-TCP

BMP2

$\mathrm{BV} / \mathrm{TV}$

CalV/TV

Cn.MARd $\beta$ beta-tricalcium phosphate

bone morphogenetic protein 2

bone volume

calcified tissue volume

trabecular mineral apposition rate at distance

${ }^{3}$ Author to whom any correspondence should be addressed.

Cn.MARn

HyA

IM

LHyA

MatV/TV

RHyA

SEM

TRAcP

VOI trabecular mineral apposition rate in new bone hyaluronic acid intra muscular linear hyaluronic acid material volume reticulated hyaluronic acid scanning electron microscopy tartrate resistant acid phosphatase volume of interest 


\section{Introduction}

Granules or particles of bone or synthetic biomaterials have been extensively used as bone substitutes in the repair of bone defects. These forms of biomaterial should set in the bony defect by filling all the void space. They should favor osteoconduction and growth of vascular sprouts while offering free access to body fluids and cells. Calcium orthophosphate biomaterials represent a large class of synthetic ceramics and have been used in the dental field for more than 20 years (Dorozhkin 2012). Beta-tricalcium phosphate ( $\beta$-TCP, $\left.\beta-\mathrm{Ca}_{3}\left(\mathrm{PO}_{4}\right)_{2}\right)$ is a suitable biodegradable calcium orthophosphate that can be prepared in various forms by a number of suppliers. Granules of suitable size can be prepared using polyurethane foam technology (Schwartzwalder et al 1963). Briefly, small cubes of polymer foam are used as templates and filled under vacuum with slurry prepared with $\beta$-TCP mixed with distilled water. Blocks are then dried, sintered at a high temperature (which destroys the foam) thus leaving porous blocks of sintered $\beta$-TCP that can be then be crushed in a mortar to give calibrated granules after sieving. It has been shown that a highly concentrated slurry produces more massive granules after sintering than when a lower amount of $\beta$-TCP in the slurry is used to infiltrate the foam (Filmon et al 2009). The size and geometry of biomaterial granules are considered critical parameters to favor bone formation (Ghanaati et al 2010, Ripamonti 1996). $\beta$-TCP particles exhibit a special conformation with a macroporosity (due to cavities of the foam) and microporosity (due to sublimation of the organic foam during sintering). In addition, $\beta$-TCP granules present surface irregularities and lateral extensions which tend to move the particles away from each other when stacked. This creates an inter-granular porosity which may also have a considerable biological role. These different types of porosity can control the access of the grafted area to macrophages, vascular sprouts and osteoprogenitor cells. However, $\beta$-TCP granules, when dry, are difficult to handle by the surgeon who often mixs them with the patient's blood to obtain a kind of paste which is easier to manage (Chappard et al 2010). The 3D disposition of the $\beta$-TCP granules (and the surrounding blood clot) in the grafted area is poorly understood although it can influence the biological response. It would be of interest to develop special formulation of a synthetic or natural hydrogel that could be used as a sealant or carrier for replacing the hazardous handling of the patient's blood. Hyaluronic acid (HyA) is a polymer of repeating disaccharide units (D-glucuronic acid and N-acetyl-D-glucosamine) encountered in various tissues (vitreous body of the eye, extra cellular matrix of cartilage, skin and also in the synovial fluid). HyA is biocompatible and is already approved for the filling of facial wrinkles or intra-articular injection in osteoarthritic joints (American College of Rheumatology Subcommittee on Osteoarthritis Guidelines 2000, Duranti et al 1998). In the present study, we investigated the effect of two types of HyA (linear or reticulated) as an aqueous binder of $\beta$-TCP granules.
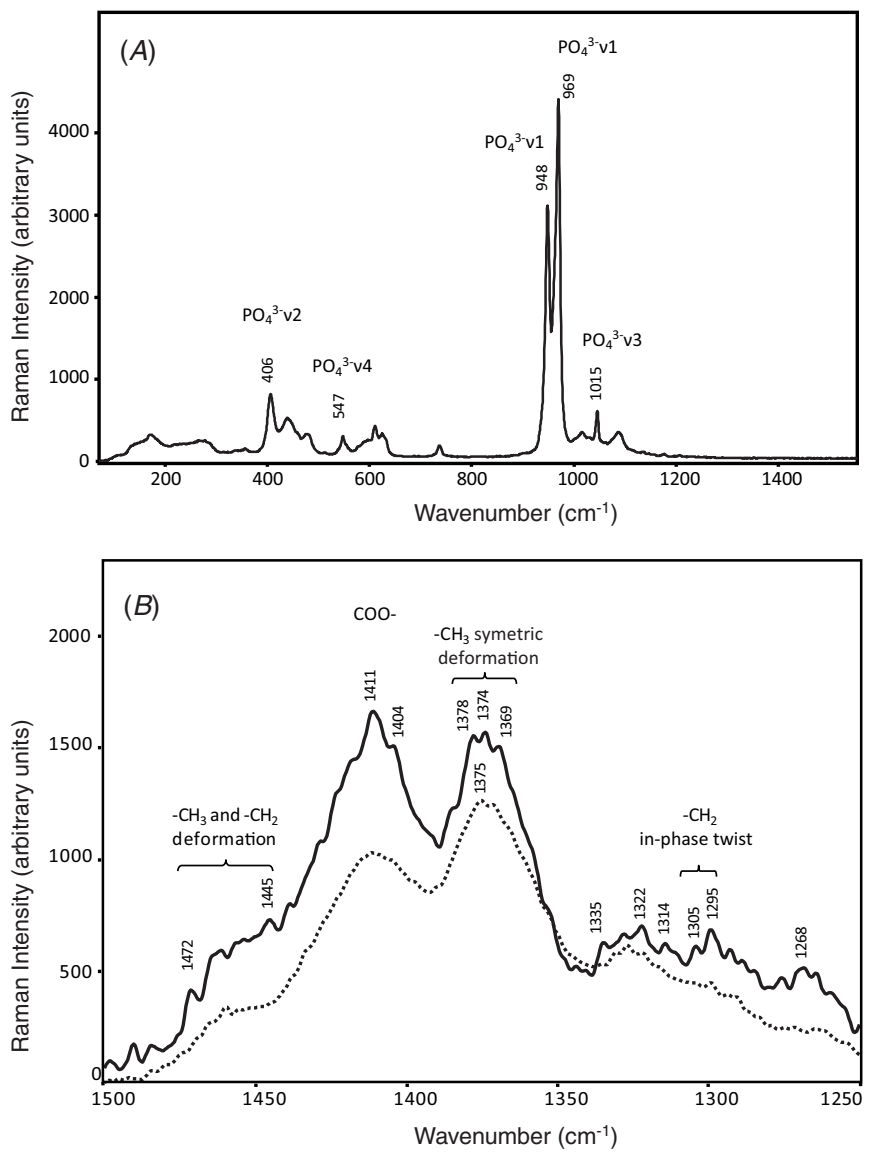

Figure 1. (A) Raman spectrum of $\beta$-TCP, locations of the different phosphate peaks are indicated. $(B)$ Raman spectra of linear and reticulated HyA. Dotted line: linear HyA, plain line: reticulated HyA.

\section{Materials and methods}

\section{1. $\beta$-TCP granules}

Granules (1000-2000 $\mu \mathrm{m}$ large) were obtained from Kasios (Launaguet, France). The morphology of granules was controlled by SEM (scanning electron microscopy) and the composition was verified by Raman microspectroscopy (figure 1(A)).

\subsection{Hyaluronic acid}

Linear HyA (LHyA) was obtained from Hyaltech (Livingston, Scotland, UK) and reticulated HyA (RHyA) from Teoxane (Geneva, Switzerland). Both HyA were provided as hydrogels in sterile syringes. According to the technical data sheets from the manufacturers, LHyA is a $10 \mathrm{mg} \mathrm{ml}^{-1}$ sodium hyaluronate solution in a phosphate buffer. RHyA is prepared by cross linking with the epoxide 1,4-butanediol diglycidylether and is provided as a $20 \mathrm{mg} \mathrm{ml}^{-1}$ solution (Teosyal First Lines ${ }^{\mathrm{TM}}$ ). Chemical formulae of these two types of HyA are presented in figure 2.

\subsection{Raman microspectroscopy}

Raman analysis of $\beta$-TCP, LHyA and RHyA were performed on a Senterra microscope with the OPUS 5.5 software (Bruker 

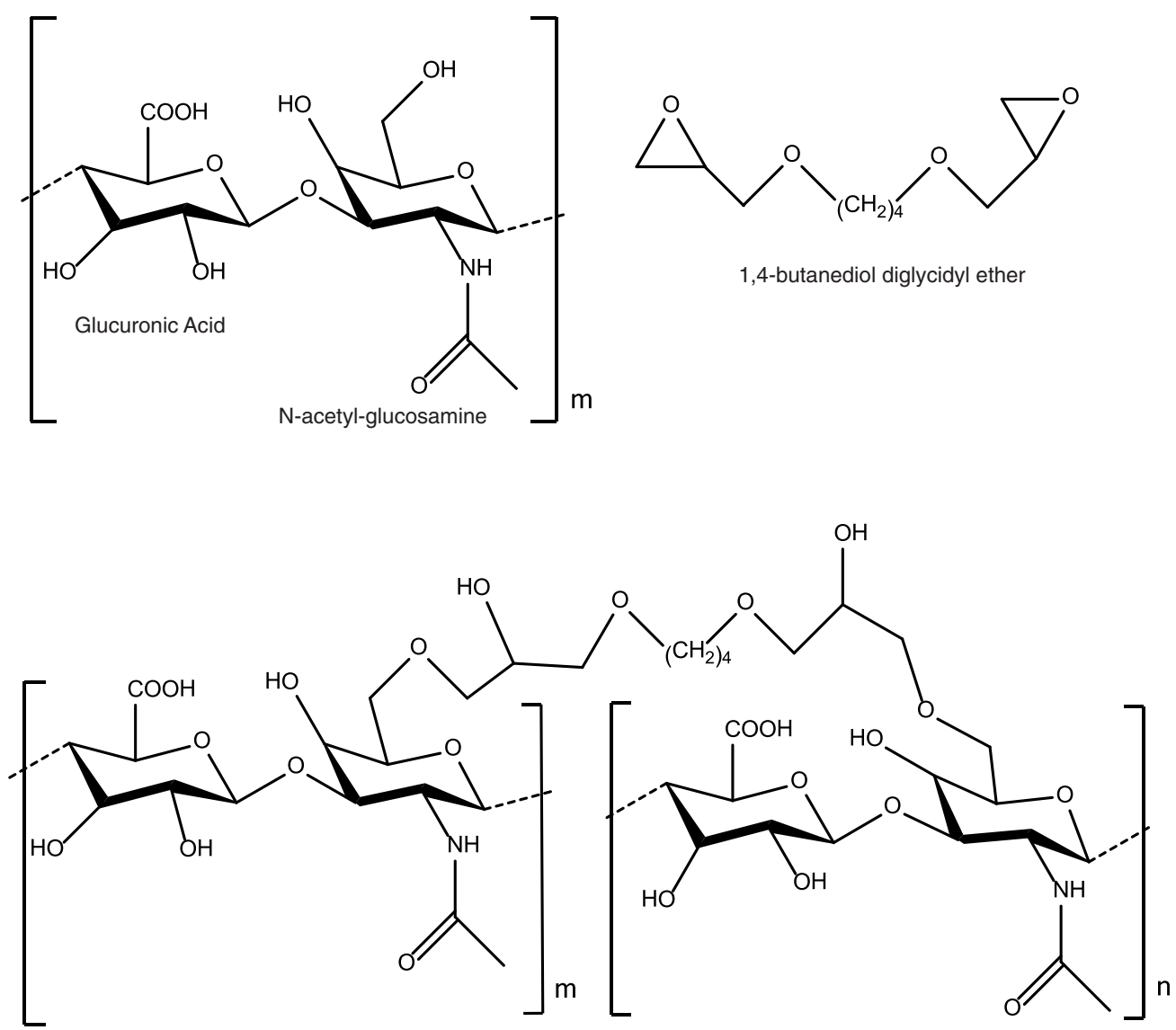

2 chains of reticulated hyaluronic acid

Figure 2. Hyaluronic acid formulations in the linear and reticulated states with 1,4-butanediol diglycidylether used as the cross-linker.

optic, Ettlingen). The excitation laser wavelength was $532 \mathrm{~nm}$ with an excitation power of $25 \mathrm{mw}$ and $3-5 \mathrm{~cm}^{-1}$ resolution. The final spectrum was obtained by averaging five scans of $20 \mathrm{~s}$ on each materials. An automatic baseline was applied. Peak intensities and bandwidth were used as described in the literature using the vendor supplied script. For $\beta$-TCP, granules were placed dry on a glass stub and imaged. For HyA, films were obtained after drying $0.1 \mathrm{~g}$ of each hydrogel in a $32{ }^{\circ} \mathrm{C}$ oven. They were transferred onto a glass slide and analysed as above.

\subsection{Animals and surgical procedure}

Thirteen New Zealand rabbits (15-16 week old and weighing approximately $3.5-3.75 \mathrm{~kg}$ ) were used in the present study. They were purchased from Charles River (L'Arbresle, France) and acclimated during eight days to the local stabling conditions. They received synthetic food and water ad libitum. Care and stabling of laboratory animals were done at ONIRIS, the Nantes veterinary school, and all procedures were approved by the local ethical comittee. Surgery was performed by trained surgeons in compliance with French law on animal experimentation. The design of the study was prepared according to the $3 \mathrm{Rs}$ recommendations concerning the number of animals (Richmond 2002). Animals were operated under general anesthesia with medetomidine (Domitor $®$, Pfizer, Paris France) and ketamine (Imalgène $1000^{\circledR}$, Merial SAS,
Villeurbanne, France). The distal femoral extremity of each femur was processed simultaneously during the same surgary. After skin incision, and lateral arthrotomy approach via the knee joint, a cylindrical defect $(4 \mathrm{~mm}$ in diameter and $6 \mathrm{~mm}$ long) was created on the outer femoral condyle with a surgical electrical driller (Acculan 3Ti: B Braun, BoulogneBillancourt) operating at low speed (200 rpm). After flushing with saline to eliminate the bone and marrow debris, the hole was filled with one of the following compositions.

(a) Linear hyaluronic acid alone was injected with the readyto-use disposable syringe provided by the manufacturer until the hole was completely filled. Three condyles were injected, constituting the LHyA group.

(b) Reticulated hyaluronic acid alone was injected similarly with the manufacturer syringe until the hole was completely filled. Four condyles were injected, constituting the RHyA group.

(c) $\beta$-TCP granules were gently pushed in the hole where they were progressively impregnated by the blood coming from the bone defect and completely filled the defect (six condyles, constituting the $\beta$-TCP group).

(d) $\beta$-TCP granules extemporaneously mixed with LHyA to prepare a viscous paste $(1.85 \mathrm{v} / \mathrm{v})$. The paste was pushed into the hole with a spatula and completely filled it (six condyles constituting the $\beta$-TCP + LHyA group); 
(e) $\beta$-TCP granules extemporaneously mixed similarly with RHyA and similarly placed in the bone defect with a spatula (six condyles, constituting the $\beta$-TCP + RHyA group).

Incision was then closed on three layers with resorbable sutures. Implantation control was done by x-ray examination to ensure that the $\beta$-TCP granules were placed in the right position. A double calcein labeling was done to determine histodynamic parameters with the following schedule 'two days on, eight days off and two days on' (Sigma-Aldrich, $30 \mathrm{mg} \mathrm{kg}{ }^{-1}$ dose via an IM route). An additional twoday period preceded euthanasia which was done 28 days after implantation (a duration that was found long enough to obtain a satisfactory bone apposition and material erosion in a previous study) (Nyangoga et al 2010). Euthanasia was done by injecting an intracardiac overdose of sodium penthobarbital (Dolethal ${ }^{\circledR}$, Vetoquinol, France). Lower femoral extremities were immediately dissected from each animal and fixed in an alcohol-formalin based fixative (BB's) according to previously published methods (Chappard 2009).

\subsection{X-ray and microcomputed tomography}

Radiographs of the implanted bones were done ex vivo using a Faxitron x-ray system with a $12 \mathrm{~cm} \times 12 \mathrm{~cm}$ CCD camera (Edimex, Le Plessis Grammoire, France). The Skyscan 1076 $\mathrm{X}$-ray computed microtomograph (Bruker microCT, Kontich, Belgium) was used in the cone beam acquisition mode. Bones, still in the fixative to prevent desiccation, were scanned according to previously published protocol for ex vivo bones during a prolonged time at $80 \mathrm{kV}, 120 \mu \mathrm{A}$ and a $1 \mathrm{~mm}$ aluminum filter (Nyangoga et al 2010). The pixel size was fixed at $9 \mu \mathrm{m}$ and a $0.20^{\circ}$ rotation angle was applied at each step and four images were averaged at each step during a $360^{\circ}$ rotation. For each femur, a stack of 2D sections was obtained in indexed grey levels ranging from 0 to 255 (figure 3(A)). The CTAn Software (Skyscan, release 1.10.1.0) was used for measuring the bone mass and architecture in the grafted area. First, the volume of interest (VOI) was designed to analyse the biomaterial and the bone ingrowth accurately. The manufacturer provided the possibility of interactively drawing polygons on the 2D sections at the margins of the hole drilled to select the whole grafted area (figure $3(B)$ ) (Bouxsein et al 2010). Only a few polygons needed to be drawn (e.g. on the first section, several at the middle, and on the final section) since a routine facility calculated all the intermediary masks by interpolation. When occasionaly a granule may have crossed the border of the hole just after implantation, the fraction outside the hole was not taken into account. $3 \mathrm{D}$ reconstructions showed that the VOI obtained with this method had the shape of a cylinder. The VOI comprised only trabecular bone, biomaterial and the marrow cavity. For the whole series of bones, the thresholds were chosen interactively by matching the greyscale visual impression to the one from the binarized image. A first threshold was determined to eliminate background noise and to select the $\beta$-TCP (which is the most $\mathrm{x}$-ray opaque) (figure $3(C)$ ). A second threshold was determined individually to select both biomaterial and bone, since bone alone cannot be segmented from the histogram distribution of grey levels (figure $3(D)$ ). The following parameters were measured in the VOI (TV, in $\mathrm{mm}^{3}$ ) according to the recommendations of the American Society for Bone and Mineral Research (Dempster et al 2013).

- Calcified tissue volume (CalV/TV, in \%), represented the percentage of all $\mathrm{x}$-ray opaque materials $(\beta$-TCP and trabecular bone inside the VOI).

- The material volume (MatV/TV, in \%), represented the percentage of the residual $\beta$-TCP which exhibited the highest x-ray opacity.

- The bone volume (BV/TV, in \%), was derived from $\mathrm{CalV} / \mathrm{TV}-\mathrm{MatV} / \mathrm{TV}$ and represented the amount of bone that had proliferated inside the VOI, between the $\beta$-TCP granules.

\subsection{Histology and histomorphometry}

Femurs were embedded undecalcified in poly (methyl methacrylate). They were cut-dry (7 $\mu \mathrm{m}$ in thickness) on a heavy-duty microtome equipped with tungsten carbide knives (Leica Polycut S-Rueil Malmaison, France). Sections were stained with a modified Goldner's trichrome. Osteoclasts were identified by a histochemical detection of tartrate resistant acid phosphatase (TRAcP). All histological techniques have been extensively described elsewhere (Chappard 2009). Histodynamic measurements done on a semiautomatic system included the trabecular mineral apposition rates in bone at distances from the implantation site $\left(\mathrm{Cn} \cdot \mathrm{MAR}_{d}\right.$, in $\left.\mu \mathrm{m} / \mathrm{d}\right)$ and in the new bone apposed on the biomaterial particles $\left(\mathrm{Cn} \cdot \mathrm{MAR}_{n}\right.$, in $\left.\mu \mathrm{m} / \mathrm{d}\right)$.

\subsection{Statistical analysis}

Statistical analysis was done using the Systat statistical software, release 13 (Systat, San José, CA). All results are expressed as mean \pm standard deviation (SD). Because of the small number of bone samples used in this study and the fact that parametric assumptions were not met, a Kruskall-Wallis non-parametric analysis of variance was used to compare the differences between the groups (Kitchen 2009). Comparison between groups was obtained by the ConoverInman post-hoc test. Differences were considered significant at $p<0.05$. Because microCT measurements are based on an interactively determined thresholds on each bone, the intraobserver agreement was determined by computation of the coefficient of variation (ratio of SD and arithmetic mean as percentage) for $\mathrm{CalV} / \mathrm{TV}$ and $\mathrm{MatV} / \mathrm{TV}$ as well as for the threshold value. To evaluate the influence of changing the thresholds by \pm 5 grey levels, the same series of ten condyles was re-analysed by using the same ROI. Measurements were reproduced by the same pathologist (after having reseted ROI and grey level threshold) in ten femoral condyles grafted with $\beta$-TCP granules with or without HyA.

\section{Results}

\subsection{Raman analysis}

Raman spectra of the two types of HyA in the 1250$1500 \mathrm{~cm}^{-1}$ wavelength are shown in figure $1(B)$ where the 

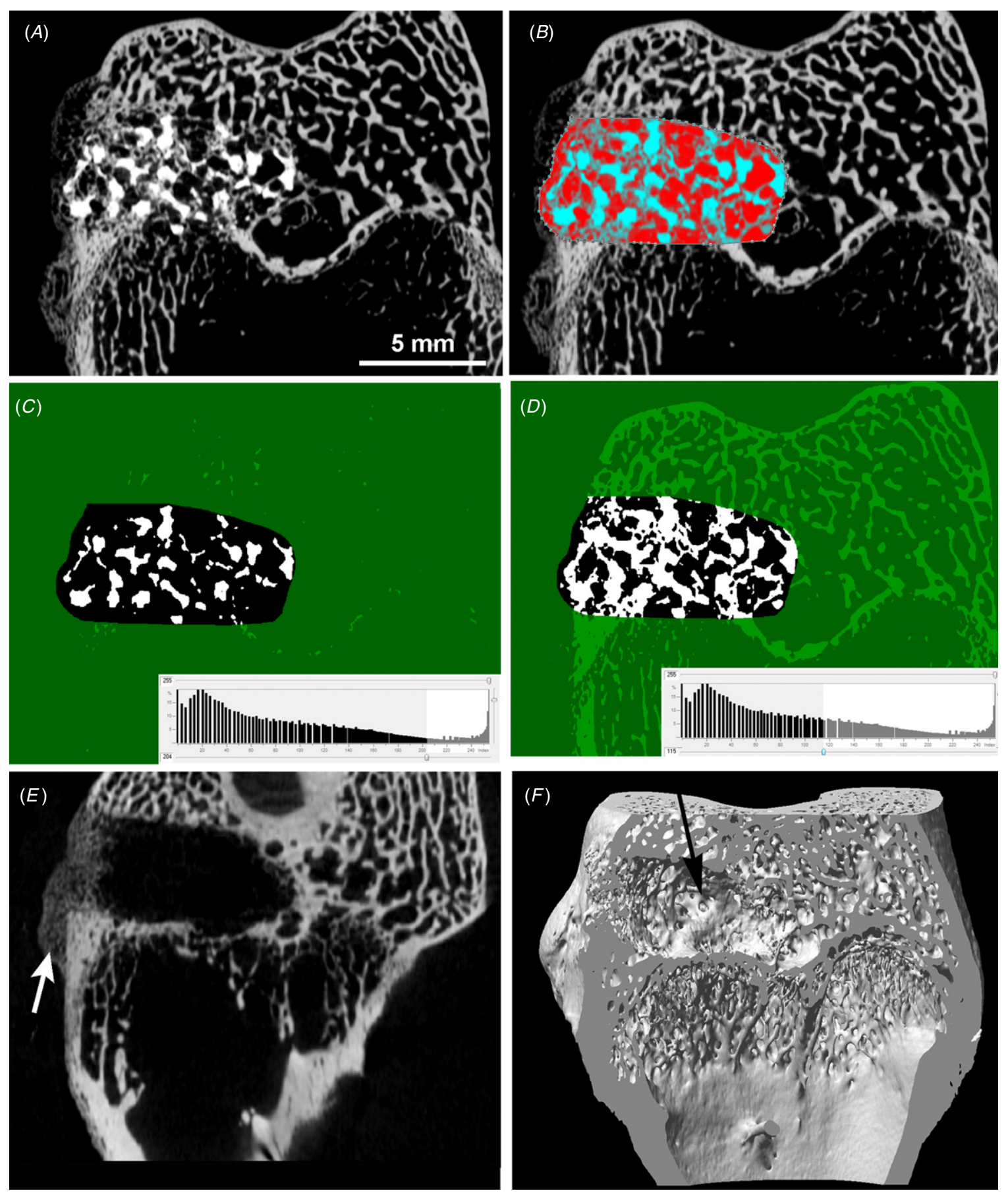

Figure 3. $(A)-(D)$ MicroCT analysis of bones with a drilled hole filled with $\beta$-TCP + RHyA. The white bar stands for $5 \mathrm{~mm}$, all $2 \mathrm{D}$ images are at the same magnification. (A) Frontal section showing the grafted area containing $\beta$-TCP granules in white with the newly formed trabeculae between the granules (in grey). Note the trabeculae from the epiphysis and metaphysis in grey. (B) A polygon drawn to limit the VOI; it follows the margins of the hole drilled in the femoral condyle. The small dots are nodes of the polygone, the surface of the polygone is in red; bone and granules appear with a blue pseudo-color. $(C)$ Same area after thresholding for the $\beta$-TCP granules. Histogram of the grey levels of image $(B)$ is figured at the bottom; the threshold is set with the lower slider (here, between 204 and 255). Note that only pixels in the VOI are selected and white-painted. (D) Same area after thresholding for the $\beta$-TCP + bone trabeculae. The threshold is now positioned between 115 and 255. (E) MicroCT analysis of a bone with a drilled hole filled with RHyA alone. The trabecular microarchitecture has not been repaired but the cortex has a large callus made of woven bone (white arrowhead). $(F)$ 3D reconstruction of the grafted area of the same animal (black arrow). Note the remodeling at the margins of the hole.

main assignment of Raman is figured. This region is the most concerned by changes related to crosslinks of the RHyA molecule induced by 1,4-butanediol diglycidyl ether which confer numerous $-\mathrm{CH}_{2}-$ to this molecule. The $-\mathrm{COO}^{-}$groups appear at $1411 \mathrm{~cm}^{-1}$ and the cross-linker induced a shift at
$1404 \mathrm{~cm}^{-1}$ (Daar et al 2010, Synytsya et al 2011). The $-\mathrm{CH}_{2}-$ peaks from the cross linking agent are evidenced in the two regions $1472-1445 \mathrm{~cm}^{-1}$ and also at $1295-1305 \mathrm{~cm}^{-1}$. The $1369-1378 \mathrm{~cm}^{-1}$ region is attributed to the $-\mathrm{CH}_{3}$ symetric deformation. 


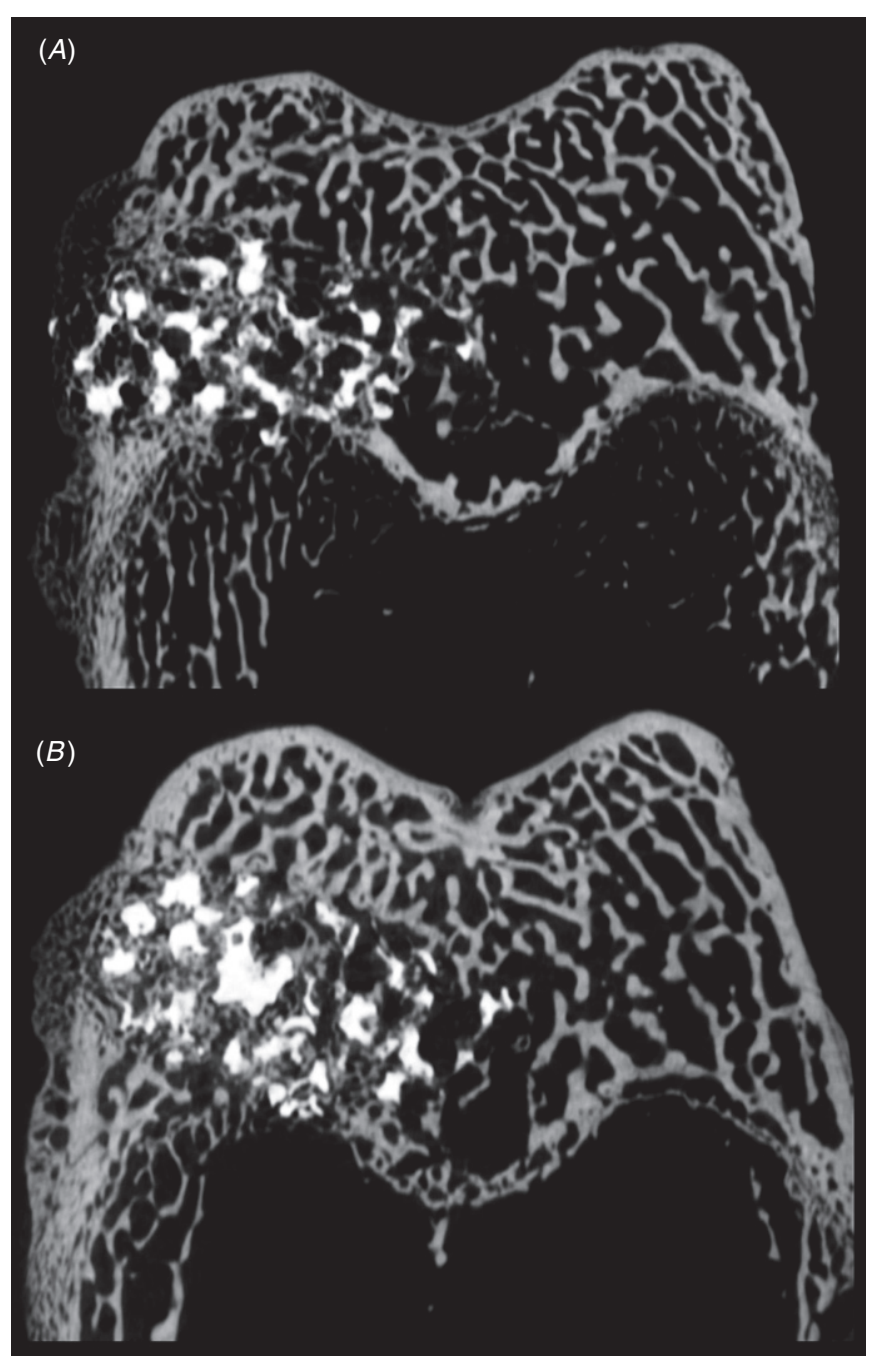

Figure 4. MicroCT analysis of bones with a drilled hole filled with $\beta$-TCP + LHyA $(A)$ and with $\beta$-TCP + RHyA $(B)$. Note that biomaterial granules are easily differentiated (in white) from bone (in grey) by their high radio-opacity. The extensive osteoconduction can be seen with numerous thin trabeculae between, and anchored, onto the granules.

\subsection{Clinical findings}

LHyA, either used alone or combined with the biomaterial granules, appeared more rapidly washed by the blood flow at the time of surgery before the closure of the surgical site. RHyA was more stable and the biomaterial granules were easier to handle. The 'paste' remained in the drilled hole and exhibited a reduced tendency to be eluted by the blood coming out of the drilled hole.

\subsection{MicroCT analysis}

Porous granules of $\beta$-TCP were clearly identified by microCT due to a higher radio-opacity than bone (figures 3 and 4) (Chappard et al 2010, Nyangoga et al 2010). The granules have retained their general shape and the internal porosity due to the manufacture process could still be evidenced. Osteogenesis was evidenced in animals which had received $\beta$-TCP (with/without HyA) as thin trabeculae engulfed in the grafted area and starting from the margins of the hole. In contrast, condyles that have been filled with LHyA or RHyA did not cause proliferation of bone from the margins of the hole. The cortical defect created during the drilling phase had always healed with a callus developed from the periosteal margins. Intra-observer reproducibility indicated a threshold variability of $0.88 \%$ and the mean SDs of the threshold values were respectively 4.41 and 2.15 for CalV/TV and MatV/TV. The variabilities of the parameters themselves were calculated as $0.0089(0.89 \%)$ for CalV/TV and $0.0094(0.94 \%)$ for $\mathrm{MatV} / \mathrm{TV}$ showing the very good reproducibility of the results produced with the method. In order to see the influence of the interactive thresholds, the values of MatV/TV and CalV/TV were recomputed by adding or substracting five grey levels to the mean threshold value. On the whole series of grafted animals, adding five grey levels provided a $+6.8 \%$ variation for MatV/TV and $+5.3 \%$ for CalV $/ \mathrm{TV}$. When five grey levels were removed from the mean threshold, $\mathrm{a}-6.0 \%$ variation was noted for MatV/TV and $-5.2 \%$ for CalV/TV. The net volume of bone $(\mathrm{BV} / \mathrm{TV})$ derived from these parameters changed between $+4.3 \%$ and $-3.9 \%$.

CalV/TV was significantly increased in both groups in which $\beta$-TCP granules have been associated with HyA when compared to $\beta$-TCP alone ( $p<0.001$ for LHyA and RHyA). When considering the amount of residual $\beta$-TCP granules alone, MatV/TV was significantly higher in the $\beta$-TCP + LHyA group than in the $\beta$-TCP and $\beta$-TCP + RHyA groups (resp. $p=0.02$ and $p=0.03$ ).

The net amount of bone formed, $\mathrm{BV} / \mathrm{TV}$, was considerably higher in the $\beta$-TCP $+\mathrm{RHyA}$ group when compared to $\beta$-TCP and $\beta$-TCP+LHyA groups $(p<0.0001$ and $p<0.004$, respectively). $\mathrm{BV} / \mathrm{TV}$ was also significantly different in all groups receiving $\beta$-TCP, LHyA or RHyA ( $p<$ 0.0001 ) (figures 4 and 5(A)). However, the net amount of bone formed in the $\beta$-TCP + LHyA group did not differ significantly from the $\beta$-TCP group.

\subsection{Histologic analysis}

In the group grafted with either LHyA or RHyA, the hole appeared filled with normal bone marrow. In all groups, the cortical defect had healed with a callus made of woven bone (figure $6(C)$ ). Bone packets were apposed directly on the surface of $\beta$-TCP granules and thin trabeculae were found to connect the granules between each other (figure $6(A)$ ). The trabeculae appeared to develop centripedically from the bone situated in the margin of the hole. Under polarized light, the trabeculae were composed of both woven and lamellar bone. A non-inflammatory loose connective tissue (made of mesenchymal cells, sinusoid capillaries, macrophages and some mast cells) has developed between $\beta$-TCP granules and new trabeculae. Macrophages presented with a cytoplasm rich in minute particles of internalized $\beta$-TCP. A large number of cuboidal osteoblast alignments was observed on the newly formed bone and on the biomaterial granules. The TRAcP reaction identified very accurately a high number of osteoclastlike giant cells at the surface of $\beta$-TCP granules and on newly formed bone (figure $6(D)$ ). Histodynamic analysis 
(A)
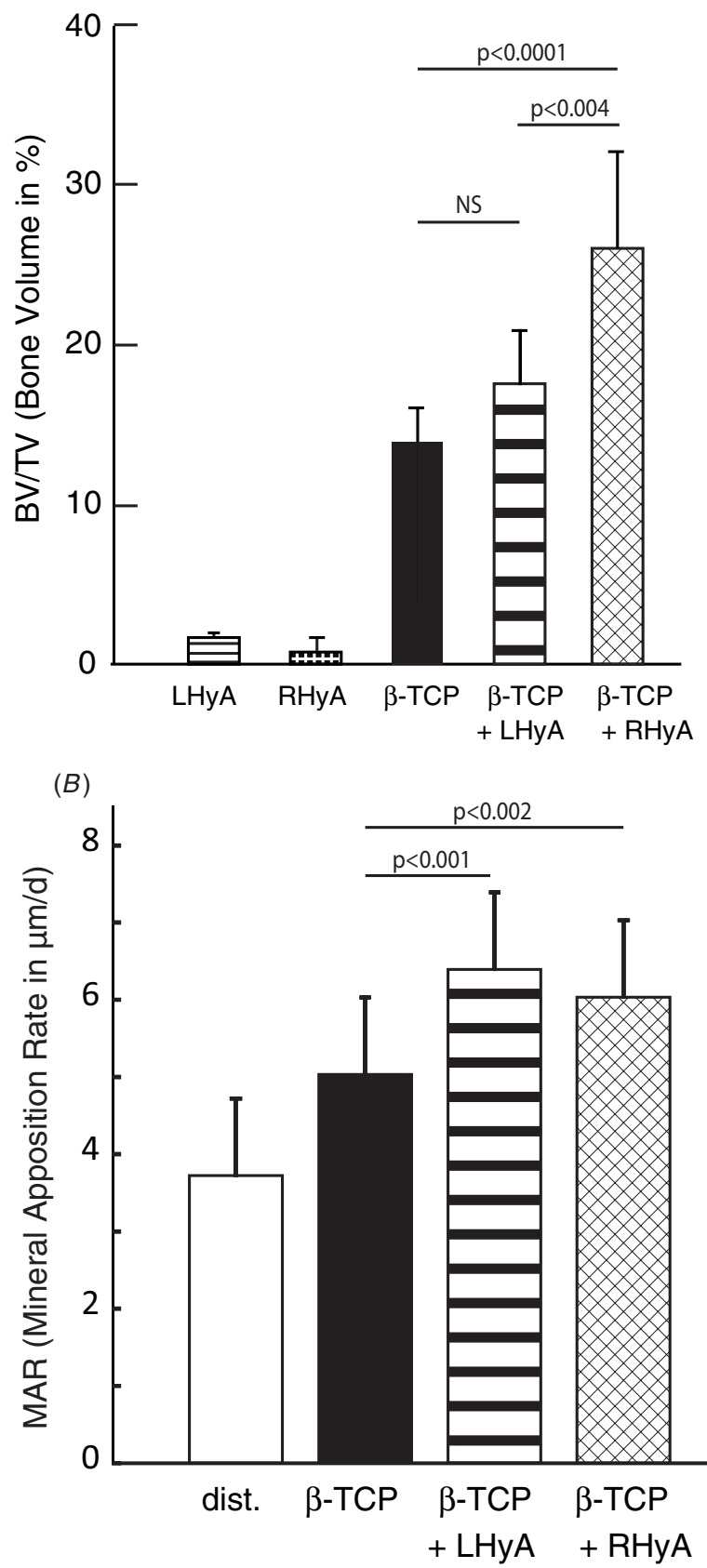

Figure 5. (A) Histomorphometric results obtained with microCT in the different grafted groups. BV/TV in the LHyA and RHya groups are significantly different from all the groups containing $\beta$-TCP.

$(B)$ Histodynamic results obtained at distance from the grafted area (dist.) and in bone trabeculae developed onto the $\beta$-TCP granules implanted alone $(\beta$-TCP) or with linear or reticulated hyaluronic acid. $\mathrm{CnMAR}_{d}$ is significantly different from all the groups with $\beta$-TCP.

(figure 6(B)) showed that $\mathrm{CnMAR}_{d}$ did not differ among groups and all values were pooled for comparison with $\mathrm{CnMAR}_{n}$ in the different groups (figure $5(B)$ ). In the areas grafted with $\beta$-TCP, $\mathrm{CnMAR}_{n}$ was considerably stimulated when compared to $\mathrm{CnMAR}_{d}$ at distance from the grafted area. A significant difference was noted between the $\beta$-TCP group and the two groups in which HyA was used.

\section{Discussion}

The study of a bone filling material necessitates the evaluation of a defect with a critical size diameter and with no spontaneous healing (Hollinger and Kleinschmidt 1990, Schmitz and Hollinger 1986). We have previously shown that a $4 \mathrm{~mm}$ hole in the femoral condyle was a critical size defect in the rabbit (Chappard et al 1993, 1995). Because of the 3Rs principle, the ethical comittee considered that performing an additional group without HyA was not necessary; also the number of animals in the RHyA and LHyA was also judged sufficient since suitable post-hoc non-parametric tests were used (Richmond 2002). Hyaluronic acid is a common natural substance of the majority of extracellular matrix; it is a radio-lucent compound. In its native form, HyA is a linear glycosaminoglycan which can bind numerous water molecules to form a hydrogel in which the chains are stabilized by weak and transient hydrogen bonds (Blundell et al 2006). These hydrogen bonds are responsible for the flexibility of the hydrogel and LHyA can retain a considerable amount of water. In this study, it was found that blood coming out of the drill hole was sufficient to elute the hydrogel thus leading to the release of some $\beta$-TCP particles. Reticuled HyA have been developed to increase the stability of the hydrogel and to limit its degradation in the body. LHyA is known to have a very short half-life of a few hours in the human body and is degraded by hyaluronidase-I (Barbucci et al 2002, Chao et al 2007). A number of cross-linkers have been proposed to reticulate HyA including carbodiidimide, glutaraldehyde or radiations. 1,4-butanediol diglycidyl ether has been proposed to prepare less toxic and hydrolyzable hydrogels suitable for cosmetic applications (Sharma and Sharma 2011, Yeom et al 2010). These more stable hydrogels are less subjected to enzyme hydrolysis but their half-life is of a few days or weeks in soft tissues (Bergeret-Galley 2004, Lemperle et al 2003, Yeom et al 2010). In the present study, Raman analysis was used to confirm the cross-links obtained in a commercial RHyA preparation used for tissue augmentation in cosmetology (Smith et al 2010, Yan et al 2009). Although the degree of cross-linking is low according to the manufacturer, the Raman signature is evidenced by a shift of the $-\mathrm{COOH}$ groups and the appearance of $-\mathrm{CH}_{2}-$ signals due to the cross links.

MicroCT is a powerful method to investigate the healing of bone defects filled with biomaterials. Because $\beta$-TCP is much more radio-opaque than bone, it is easy to quantify the respective amounts of biomaterial and bone in a reference volume (Chappard et al 2010, Nyangoga et al 2010).

When injected in the drilled hole, LHyA and RHyA did not cause healing of the grafted site and the trabecular microarchitecture was not restored. BV/TV remained very low and only the margins of the hole showed a limited remodeling. On histological sections, the bone marrow was reconstituted without signs of inflammation. No medullary edema was noted. There was no sign of extracellular material even on sections stained with toluidine blue. Because injection of RHyA in soft tissues has been shown to be associated wih remnants of the material wihin 2-3 months, clearly this 


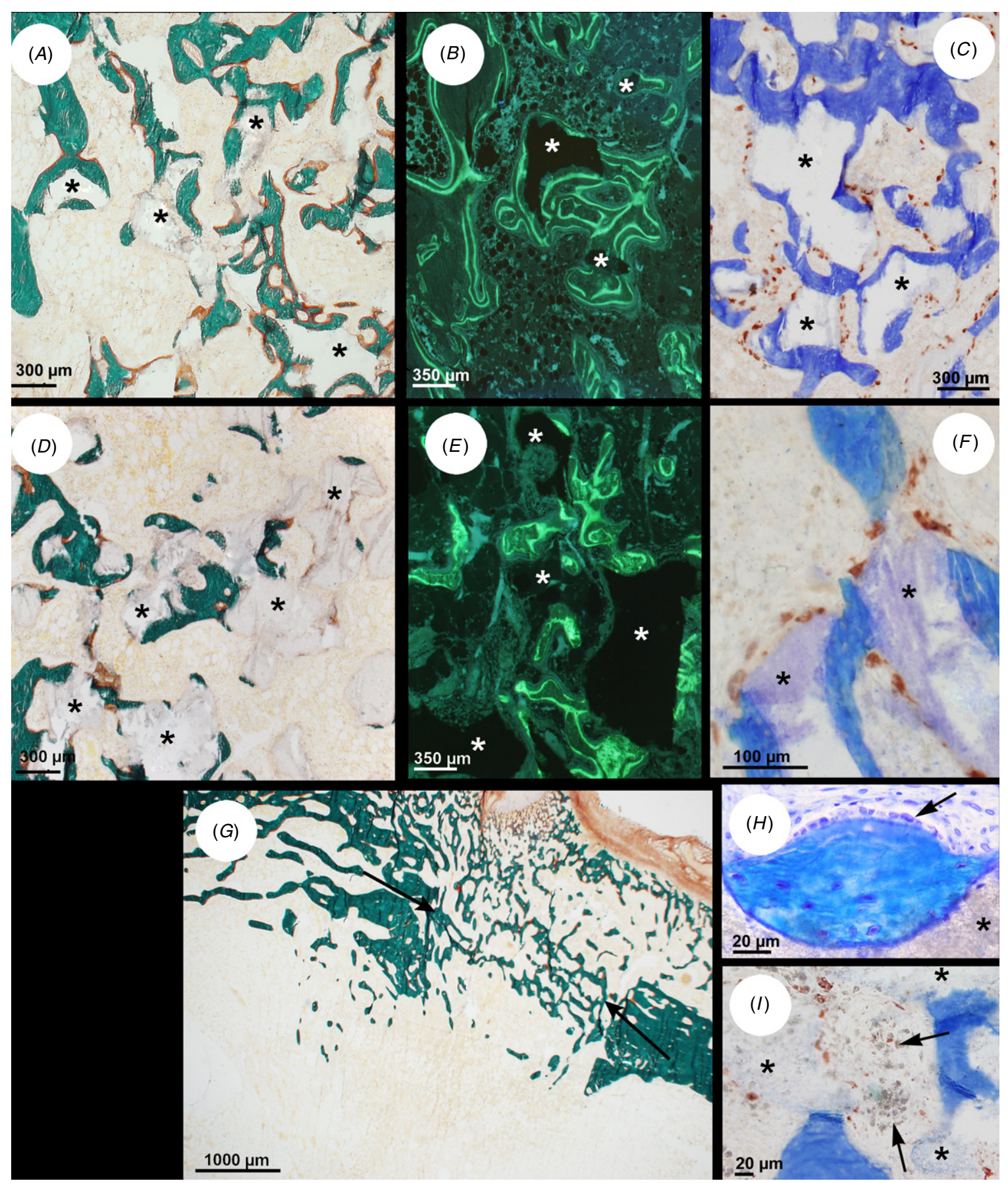

Figure 6. Histological analysis of undecalcified bone sections of femoral condyles; on each image the $\beta$-TCP granules are identified by a * (A) Rabbit grafted with $\beta$-TCP granules RHyA (Goldner stain). Note the extension of thin and mineralized trabeculae (in green) composed of woven and lamellar bone and covered by osteoid seams (in red); bone marrow is in light yellow. (B) Same animal, double calcein labeling. Note the large width between the two bands on the bone deposited on $\beta$-TCP granules. $(C)$ Histochemical identification of osteoclast-like giant cells by TRAcP staining. These cells are present at the surface of $\beta$-TCP granules and on the newly deposited bone; positive cells are in brown, bone is counter-stained in blue; other cells are unstained. (D) Rabbit grafted with $\beta$-TCP granules LHyA (Goldner stain). Mineralized bone is apposed onto the granules but the amount of newly formed bone is less important than with RHyA. (E) Same animal, double calcein labeling with numerous double labels. (F) Same animal, histochemical identification of osteoclasts by TRAcP staining at a higher magnification than in $(C) .(G)$ low magnification of the drilled hole from a rabbit grafted with RHyA alone, the periosteal callus is evidenced (arrow) with no bone restauration in the marrow, Goldner's trichrome. $(H)$ A bone packet (in blue) containing osteocytes is directly apposed on a $\beta$-TCP granule by a layer of osteoblasts (arrow). Toluidine blue staining. (I) Histochemical identification of osteoclasts by TRAcP staining in a rabbit having received $\beta$-TCP granules RHyA. Osteoclasts are identified by their brown tint. Numerous macrophages with a cytoplasm filled with small particules of eroded $\beta$-TCP are present inside the loose connective tissue developed between the granules and the trabeculae (arrows). 
was not the case in our study. This can be due to the high amount of macrophages present within the bone marrow which induce a rapid destruction of the hydrogel (Tamaki et al 2008). When L- or RHyA were used to handle the $\beta$-TCP particles, significantly more granules were deposited in the drilled hole even with the rapidly eluted LHyA. RHyA permitted to keep up the granules in the site. Consequently, this induced the deposit of significantly more bone. The $\beta$-TCP granules were more numerous with L- or RHyA thus leading to a significant activation of bone remodeling as evidenced by the increase in $\mathrm{CnMAR}_{n}$ in these groups when compared with the $\beta$-TCP group. However, due to the short half-life of these HyA hydrogels and their complete disappearance in histological sections, it is likely that the $3 \mathrm{D}$ arrangement of the granules is the key factor for bone remodeling stimulation. In in vitro conditions, HyA has been shown to increase osteoblast activity (Huang et al 2003). In addition, although several authors have reported that osteogenic factors such as BMP2 can be trapped by $\mathrm{HyA}$, the complete restoration of the bone marrow without bone development in the LHyA and RHyA groups is not in favor of this hypothesis (Kim and Valentini 2002). Furthermore, BMP2-HyA gels have been found to have no effect on bone remodeling (Maus et al 2008).

Several types of hydrogels have been proposed to help $\beta$-TCP granules implantation: methylcellulose but it can cause inflammatory reactions (Ghanaati et al 2011); methylcellulose cross-linked with silane groups (Fellah et al 2006, Isaji and Naito 1992) and hyaluronic acid (Chazono et al 2004). The use of the natural biopolymer HyA was found to enhance bone apposition in the grafted areas although the mechanisms were not clearly evaluated (Aslan et al 2006).

\section{Conclusion}

Reticulated HyA provided a suitable medium to deposit a larger amount of $\beta$-TCP granules in a bone defect. The elution of RHyA from the bone defect by blood or extracellular fluids allows an easy handling of the $\beta$-TCP granules. The rapid disappearance of the hydrogel without secondary inflammatory reaction is an asset for the use of such commercially available hydrogels.

\section{Acknowledgments}

This work was made possible by grants from Contrat de Plan Etat-Région 'Pays de la Loire' Bioregos2. The $\beta$-TCP was provided by Kasios (Launaguet, France). Thanks to Mrs L Lechat for secretarial assistance, to Mrs Izabela Stancu (University Polythenica of Bucharest) for her help with the chemical formulae and to Mrs Laurence Hermitte for technical assistance with HyA formulations.

The authors declare that they have no conflict of interest.

\section{References}

American College of Rheumatology Subcommittee on Osteoarthritis Guidelines 2000 Recommendations for the medical management of osteoarthritis of the hip and knee: 2000 update Arthritis Rheum. 43 1905-15
Aslan M, Simsek G and Dayi E 2006 The effect of hyaluronic acid-supplemented bone graft in bone healing: experimental study in rabbits J. Biomater. Appl. 20 209-20

Barbucci R, Lamponi S, Borzacchiello A, Ambrosio L, Fini M, Torricelli P and Giardino R 2002 Hyaluronic acid hydrogel in the treatment of osteoarthritis Biomaterials 23 4503-13

Bergeret-Galley C 2004 Comparison of resorbable soft tissue fillers Aesthet. Surg. J. 24 33-46

Blundell C D, Deangelis P L and Almond A 2006 Hyaluronan: the absence of amide-carboxylate hydrogen bonds and the chain conformation in aqueous solution are incompatible with stable secondary and tertiary structure models Biochem. $J$. $396487-98$

Bouxsein M L, Boyd S K, Christiansen B A, Guldberg R E, Jepsen K J and Muller R 2010 Guidelines for assessment of bone microstructure in rodents using micro-computed tomography J. Bone Miner. Res. 25 1468-86

Chao K L, Muthukumar L and Herzberg O 2007 Structure of human hyaluronidase-1, a hyaluronan hydrolyzing enzyme involved in tumor growth and angiogenesis Biochemistry 46 6911-20

Chappard D 2009 Technical aspects: How do we best prepare bone samples for proper histological analysis? Bone Cancer: Progression and Therapeutic Approaches ed D Heymann (New York: Academic/Elsevier) pp 203-10

Chappard D, Baslé M F, Grizon F and Rebel A 1995 Purified xenogeneic bone bused as a bone substitute (1): comparison of the healing of bone defects filled with autografts, hydroxylapatite or a xenogeneic grafts (T650)—an in vivo study in the rabbit femoral condyle Biomed. Res. 6 211-22

Chappard D, Guillaume B, Mallet R, Pascaretti-Grizon F, Baslé M F and Libouban H 2010 Sinus lift augmentation and beta-TCP: a microCT and histologic analysis on human bone biopsies Micron 41 321-6

Chappard D, Zhioua A, Grizon F, Baslé M F and Rebel A 1993 Biomaterials for bone filling: comparisons between autograft, hydroxyapatite and one highly purified bovine xenograft Bull. Assoc. Anat. 77 59-65

Chazono M, Tanaka T, Komaki H and Fujii K 2004 Bone formation and bioresorption after implantation of injectable beta-tricalcium phosphate granules-hyaluronate complex in rabbit bone defects J. Biomed. Mater. Res. A 70 542-9

Daar E, King L, Nisbet A, Thorpe R B and Bradley D A 2010 Viscosity changes in hyaluronic acid: irradiation and rheological studies Appl. Radiat. Isot. 68 746-50

Dempster D W, Compston J E, Drezner M K, Glorieux F H, Kanis J A, Malluche H, Meunier P J, Ott S M, Recker R R and Parfitt A M 2013 Standardized nomenclature, symbols, and units for bone histomorphometry: a 2012 update of the report of the ASBMR histomorphometry nomenclature committee J. Bone Miner. Res. 28 2-17

Dorozhkin S V 2012 Biphasic, triphasic and multiphasic calcium orthophosphates Acta Biomater. 8 963-77

Duranti F, Salti G, Bovani B, Calandra M and Rosati M L 1998 Injectable hyaluronic acid gel for soft tissue augmentation. A clinical and histological study Dermatol. Surg. 24 1317-25

Fellah B H, Weiss P, Gauthier O, Rouillon T, Pilet P, Daculsi G and Layrolle P 2006 Bone repair using a new injectable self-crosslinkable bone substitute J. Orthop. Res. 24 628-35

Filmon R, Retailleau-Gaborit N, Brossard G, Grizon-Pascaretti F, Baslé M F and Chappard D 2009 Preparation of $\beta$-TCP granular material by polyurethane foam technology Image Anal. Stereol. 28 1-10

Ghanaati S, Barbeck M, Hilbig U, Hoffmann C, Unger R E, Sader R A, Peters F and Kirkpatrick C J 2011 An injectable bone substitute composed of beta-tricalcium phosphate granules, methylcellulose and hyaluronic acid inhibits connective tissue influx into its implantation bed in vivo Acta Biomater. 7 4018-28 
Ghanaati S et al 2010 Influence of beta-tricalcium phosphate granule size and morphology on tissue reaction in vivo Acta Biomater. 6 4476-87

Hollinger J O and Kleinschmidt J C 1990 The critical size defect as an experimental model to test bone repair methods J. Craniofac. Surg. 1 60-8

Huang L, Cheng Y Y, Koo P L, Lee K M, Qin L, Cheng J C and Kumta S M 2003 The effect of hyaluronan on osteoblast proliferation and differentiation in rat calvarial-derived cell cultures J. Biomed. Mater. Res. A 66 880-4

Isaji M and Naito J 1992 Comparative studies on inflammatory reactions induced by non-immunological and immunological stimuli in an air pouch and in a carboxymethyl cellulose (CMC)-induced inflammatory pouch Int. J. Exp. Pathol. 73 231-9

Kim H D and Valentini R F 2002 Retention and activity of BMP-2 in hyaluronic acid-based scaffolds in vitro J. Biomed. Mater. Res. 59 573-84

Kitchen C M 2009 Nonparametric versus parametric tests of location in biomedical research Am. J. Ophthalmol. 147 571-2

Lemperle G, Morhenn V and Charrier U 2003 Human histology and persistence of various injectable filler substances for soft tissue augmentation Aesthetic. Plast. Surg. 27 354-67

Maus U, Andereya S, Gravius S, Siebert C H, Ohnsorge J A and Niedhart C 2008 Lack of effect on bone healing of injectable BMP-2 augmented hyaluronic acid Arch. Orthop. Trauma Surg. 128 1461-6

Nyangoga H, Aguado E, Goyenvalle E, Baslé M F and Chappard D 2010 A non-steroidal anti-inflammatory drug (ketoprofen) does not delay beta-TCP bone graft healing Acta Biomater. 6 3310-7

Richmond J 2002 Refinement, reduction, and replacement of animal use for regulatory testing: future improvements and implementation within the regulatory framework ILAR J. $\mathbf{4 3}$ (Suppl.) S63-8

Ripamonti U 1996 Osteoinduction in porous hydroxyapatite implanted in heterotopic sites of different animal models Biomaterials 17 31-5

Schmitz J P and Hollinger J O 1986 The critical size defect as an experimental model for craniomandibulofacial nonunions Clin. Orthop. Relat. Res. 205 299-308

Schwartzwalder K, Somers H and Somers A V 1963 Method of making porous ceramics US Patent 3, 090, 094

Sharma P and Sharma S 2011 Comparative study of a new dermal filler Uma Jeunesse ${ }^{\circledR)}$ and Juvederm ${ }^{\circledR}$ J. Cosmet. Dermatol. $10118-25$

Smith S R, Jones D, Thomas J A, Murphy D K and Beddingfield F C III 2010 Duration of wrinkle correction following repeat treatment with Juvederm hyaluronic acid fillers Arch. Dermatol. Res. 302 757-62

Synytsya A, Synytsya A, Petr A, Wagner R, Davídkov M and Volkaa K 2011 Raman spectroscopic study on sodium hyaluronate: an effect of proton and $\gamma$ irradiation J. Raman Spectrosc. 42 544-50

Tamaki Y, Sasaki K, Sasaki A, Takakubo Y, Hasegawa H, Ogino T, Konttinen Y T, Salo J and Takagi M 2008 Enhanced osteolytic potential of monocytes/macrophages derived from bone marrow after particle stimulation J. Biomed. Mater. Res. B Appl. Biomater. 84 191-204

Yan X, Xu J, Lu C, Ma Y and Li W 2009 A multicenter study of the efficacy and safety of restylane in the treatment of nasolabial folds in China Plast. Reconstr. Surg. 124 256e-7e

Yeom J, Bhang S H, Kim B S, Seo M S, Hwang E J, Cho I H, Park J K and Hahn S K 2010 Effect of cross-linking reagents for hyaluronic acid hydrogel dermal fillers on tissue augmentation and regeneration Bioconjug. Chem. 21 240-7 